Spotlight

\section{Dynamic Histone H3 Incorporation Fuels Metastatic Progression}

Iva Simeonova1,2,3,4

and Geneviève Almouzni1,2,3,4,*

Treating metastatic cancers is an ongoing challenge in oncology. A recent paper by Gomes and colleagues proposes histone $\mathrm{H} 3$ variant dynamics as major regulator of cell fate transition during metastasis and suggests histone chaperones as therapeutic targets for invasive carcinoma.

Recent large-scale sequencing of metastatic cancers from more than 10000 patients represents a major step forward in our understanding of metastatic cancer genomes [1]. However, our knowledge about the dynamic chromatin landscape in metastatic cancers is still very limited. In a recent publication in Cancer Cell, Gomes et al. [2] provide hints into how extracellular signal regulated kinase (ERK)-dependent metastatic signaling, which is among the most frequently altered pathways in cancer progression, promotes a switch in histone $\mathrm{H} 3$ variant incorporation into chromatin to trigger an epigenomic and transcriptional reprograming towards cancer aggressiveness [2].

Histone variants, the building blocks of chromatin, mark particular regions of the genome in each cell type. This involves incorporation by distinct histone chaperones [3]. The chromatin assembly factor 1 complex (CAF-1) promotes deposition of the replicative $\mathrm{H} 3.1 / \mathrm{H} 3.2$ histone variants in a DNA-synthesis-dependent manner, mainly during replication. In contrast, the HIRA chaperone complex ensures the deposition of H3.3 throughout the cell cycle at actively transcribed regions, while the ATRX/ DAXX complex is rather involved in $\mathrm{H} 3.3$ enrichment in heterochromatin regions at telomeres and peri-centromeres. However, when H3.1 incorporation is compromised at DNA replication sites, HIRAdependent $\mathrm{H} 3.3$ deposition kicks in. This alternative $\mathrm{H} 3.3$ deposition at places of histone loss has been proposed to act as a 'gap-filling' mechanism to prevent nucleosome-free DNA accumulation and preserve chromatin stability [4].

Histone $\mathrm{H} 3$ variants play essential roles during development, cell fate determination, and cellular reprograming [3], and mutations thereof have been identified as oncohistones [5]. Epithelial mesenchymal transition (EMT), which promotes cell motility and differentiation as well as resistance to apoptotic stimuli during early development, is frequently co-opted by cancer cells to promote metastatic formation and dissemination [1,2]. Gomes and colleagues addressed how histone $\mathrm{H} 3$ variant dynamics are involved in cell reprogramming during metastatic transition [2]. In a variety of epithelial or carcinoma cell lines, after EMT and metastasis induction by using TGF- $\beta$ / TNF $\alpha$ or expression of mutant form of ERK, they observe increased chromatin accessibility and a general decrease of total histones incorporated into chromatin. This general loss of histones is reflected at the mRNA and protein levels for histone H3.1. However, in sharp contrast, under the same conditions, H3.3 incorporation into chromatin increased (Figure 1). This finding is reminiscent of the histone loss that facilitates reprogramming and 'gapfilling' by H3.3 [4]. Remarkably, instead of a random H3.3 enrichment, the authors find enrichment of $\mathrm{H} 3.3$ at specific promoters corresponding to genes involved in EMT, such as ZEB1, SNAI1, and $\mathrm{SO} \times 9$, all associated with induction of protumoral transcriptional programs, including tumor progression, metastasis, and wound healing (Figure 1).

Given the roles of CAF-1 and HIRA in escorting histone $\mathrm{H} 3$ variants for their incorporation into chromatin, the authors analyzed the levels of these histone chaperones. While CAF-1 decreased, HIRA proteins increased in response to EMT and metastasis inducers. Further analyses show that the decrease of CAF-1 levels is due to downregulation of its subunit CHAF1B mRNA expression, ultimately resulting in destabilization of the whole CAF-1 complex. This is achieved through inhibition of phosphorylation of a key CHAF1B transcriptional regulator, Sp1. In a series of gain- and loss-of-function experiments, the authors finely dissected the implication and importance of CAF-1, HIRA, and H3.3 in this metastatic reprograming. They found that reduction of CAF-1 is sufficient to induce EMTrelated gene expression in a variety of cancer cell lines and thereby confers resistance to anticancer drugs, such as paclitaxel and carboplatin. In an animal xenograft model, they further document increased tumor growth under similar conditions. Finally, the ectopic CAF-1 overexpression in an aggressive breast metastatic cell line LM2 prevented tumor growth, demonstrating that this axis represents a valuable means for therapeutic intervention. In contrast, the ectopic overexpression of H3.3, HIRA, or DAXX did not result in the acquisition of metastatic signature, namely fibronectin expression and loss of E-cadherin. Yet, reduction of either H3.3 or HIRA levels in LM2 cells by shRNA was sufficient to lose fibronectin and induce E-cadhering expression, indicating a loss of metastatic properties. Intriguingly, H3F3A overexpression, one of the two genes encoding $\mathrm{H} 3.3$, has been previously involved in promoting lung cancer cell invasion and associated with relapse and poor prognosis 


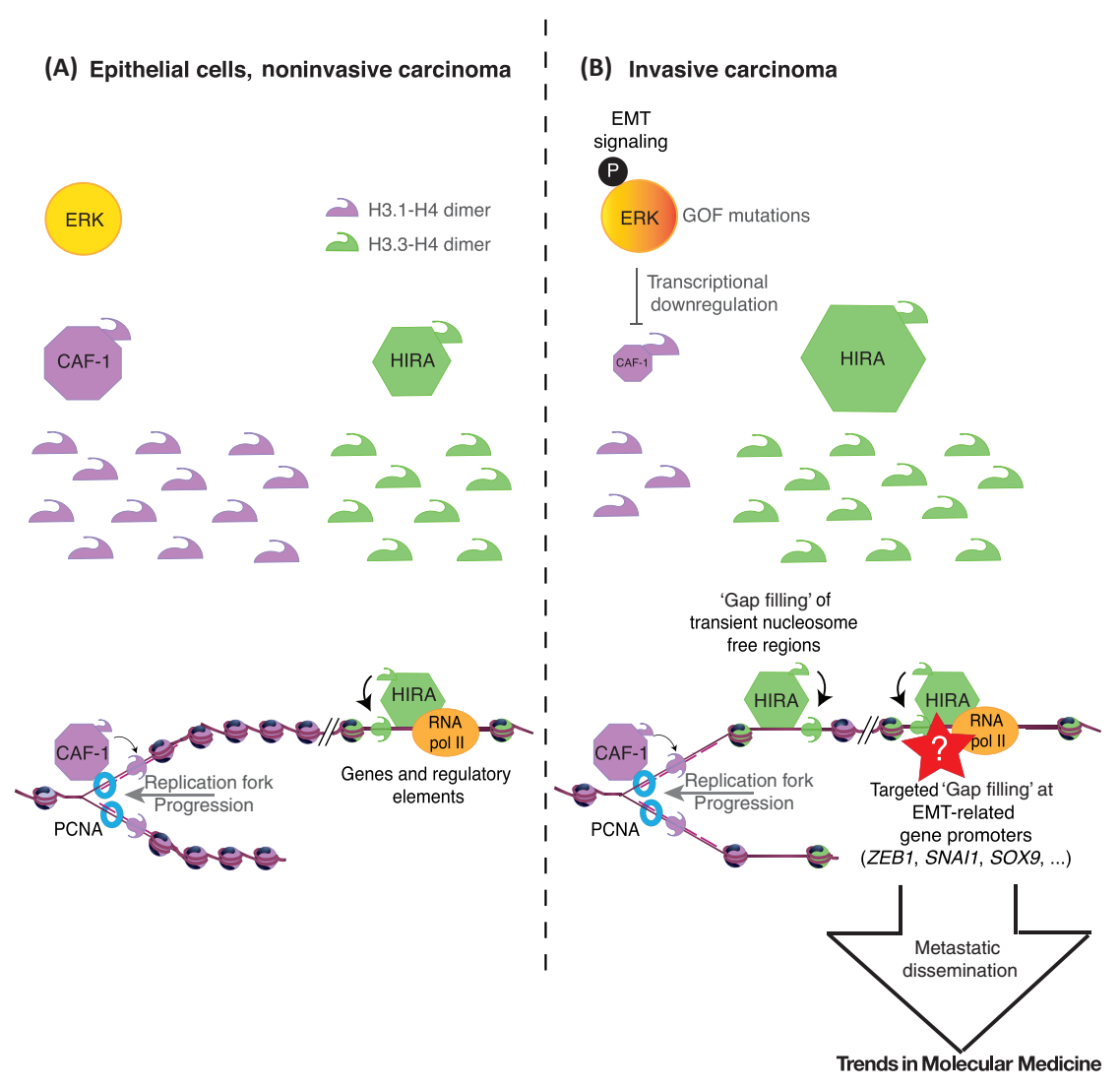

Figure 1. Histone H3 Chaperone-Dependent Chromatin Reprogramming Promotes Carcinoma Invasiveness

(A) In epithelial cells and noninvasive carcinoma, the ERK signaling pathway is not activated by oncogenic signals. The histone H3.1 (purple) is incorporated into chromatin by the chaperone CAF-1 during DNA replication, while the histone $\mathrm{H} 3.3$ variant (green) is incorporated at genes and regulatory elements by its chaperone HIRA. HIRA is also proposed to incorporate H3.3 at transient nucleosome free regions by 'gap filling', not represented here. A balance between the levels of $\mathrm{H} 3.1$ and H3.3 and their chaperones maintains the nucleosomal density at the chromatin level. (B) In invasive carcinoma, gain-of-function mutations of ERK or oncogenic stimuli leading to ERK activating phosphorylation (black circle), trigger a transcriptional downregulation of CAF-1. This is accompanied by a dramatic decrease in the histone H3.1, an increase in $\mathrm{H} 3.3$ levels (although total histone $\mathrm{H} 3$ levels remain reduced), and an increase of HIRA levels. The depletion of CAF-1 results in a reduction of $\mathrm{H} 3.1$ variant incorporated into chromatin. Gomes et al. propose a model where the arising 'naked DNA gaps' are subsequently filled in by H3.3 in a HIRA-dependent manner. HIRA and probably other unknown factors (red star) target $\mathrm{H} 3$.3-specific enrichment at promoters of EMT inducers such as ZEB1, SNAI1, and SOX9 and favors their expression. This promotes in turn a cellular transcriptional reprogramming toward a more aggressive metastatic colonization Abbreviations: CAF-1, chromatin assembly factor 1; EMT, epithelial to mesenchymal transition; ERK, extracellular signal regulated kinase; GOF, gain-of-function; PCNA, proliferating cell nuclear antigen; Sox9, sry-related high mobility group box 9; ZEB1, zinc-finger E-box binding homolog 1.

[6]. Histone H3.3 enrichment at intronic DNA regulatory elements proved critical to activate the $G$ protein-coupled receptor 87 (GPR87), acting upstream of ERK signaling [7] .
In summary, the results of Gomes and colleagues stress how ERK signaling leads to both downregulation of CAF-1 and a decrease in nucleosome density, thereby enabling cell reprog- ramming leading to resistance to anticancer therapies. This is accompanied by HIRA-dependent H3.3 incorporation at the promoters of transcription factors involved in the induction of EMT, which fuels metastatic progression. Given that HIRA reduction reverts the metastatic properties of LM2 cells, the authors put forward HIRA as a promising therapeutic target. The reduced levels of CAF-1 in metastatic breast carcinoma, when compared with their matched primary tumors, supports the findings of Gomes et al.

Previous studies have implicated CAF-1 as a barrier to embryonic stem cell reprograming, at least in part by regulating chromatin accessibility [8]. The findings of Gomes et al. imply that a similar mechanism likely occurs in tumor cells during the transition toward metastatic progression. While the H3.3 gap-filling deposition is thought to occur without DNA sequence specificity through recognition of naked DNA by HIRA, in the study by Gomes et al, HIRA brings H3.3 to specific promoters. Understanding how this specific H3.3 deposition is controlled will be exciting. Furthermore, HIRA functions as a complex and with partners, and the recent characterization of its trimerization properties offers interesting avenues to explore how this specificity could be achieved [9]. Fully understanding the role of histone $\mathrm{H} 3$ variant dynamics during metastasis will be a major future challenge, relevant not only for cancer but also for other physiological cellular reprogramming events associated with development and/or disease transition. In particular, deciphering the underlying molecular processes that control H3.3 and HIRA upregulation upon metastatic induction will be an important step to find means to prevent metastasis.

Given that such changes can affect subpopulations of cells in a tumor, new 
approaches such as fluorouracillabeled nascent RNA sequencing, designed to detect rare cell populations' trajectories in vivo and in situ [10] and single cell technologies will be instrumental to appreciate tumor heterogeneity and the transcriptional changes upon metastatic reprograming and, ultimately, to resolve the challenges imposed by metastatic divergent clonal evolution [11].

\section{Acknowledgments}

The authors thank D. Ray-Gallet and D. Jeffery for critical reading and discussion.

${ }^{1}$ Chromatin dynamics, Equipe Labellisée Ligue contre le Cancer, Institut Curie 75005, Paris,

France

${ }^{2}$ CNRS (French National Center for Scientific Research), Nuclear Dynamics unit UMR3664,

Paris, France

${ }^{3}$ Sorbonne Université 75005, Paris, France

${ }^{4}$ PSL Research University, Paris, France

${ }^{\star}$ Correspondence:

genevieve.almouzni@curie.fr

https://doi.org/10.1016/j.molmed.2019.09.003

(c) 2019 Elsevier Ltd. All rights reserved.

\section{References}

1. Zehir, A. et al. (2017) Mutational landscape of metastatic cancer revealed from prospective clinical sequencing of 10,000 patients. Nat. Med. 23, 703-713

2. Gomes, A.P. et al. (2019) Dynamic incorporation of histone $\mathrm{H} 3$ variants into chromatin is essential for acquisition of aggressive traits and metastatic colonization. Cancer Cell. https://doi.org/ 10.1016/j.ccell.2019.08.006

3. Filipescu, D. et al. (2014) Histone H3 variants and their chaperones during development and disease: contributing to epigenetic control. Annu. Rev. Cell Dev. Biol. 30, 615-646

4. Ray-Gallet, D. et al. (2011) Dynamics of histone $\mathrm{H} 3$ deposition in vivo reveal a nucleosome gap-filling mechanism for H3.3 to maintain chromatin integrity. Mol. Cell. 44, 928-941

5. Mohammad, F. and Helin, K. (2017) Oncohistones: drivers of pediatric cancers. Genes Dev. 31, 2313-2324

6. Park, S.M. et al. (2016) Histone variant H3F3A promotes lung cancer cell migration through intronic regulation. Nat. Commun. 7, 12914
7. Zhang, Y. et al. (2010) G protein-coupled receptor 87: a promising opportunity for cancer drug discovery. Mol. Cell Pharmacol. 2, 111-116

8. Yadav, T. et al. (2018) Chromatin plasticity: a versatile landscape that underlies cell fate and identity. Science 361, 1332-1336

9. Ray-Gallet, D. et al. (2018) Functional activity of the H3.3 histone chaperone complex HIRA requires trimerization of the HIRA subunit. Nat. Commun. 9, 3103

10. Basnet, H. et al. (2019) Flura-seq identifies organ-specific metabolic adaptations during early metastatic colonization. Elife 8, e43627

11. Lawson, D.A. et al. (2018) Tumour heterogeneity and metastasis at single-cell resolution. Nat. Cell Biol. 20, 1349-1360

\section{Spotlight}

\section{Boosting Antitumour Immunity through Targeted Delivery of Interferon- $\alpha$}

\author{
Paul J. Fairchild ${ }^{1, *}$ \\ and Timothy J. Davies ${ }^{1}$
}

Immune checkpoint inhibitors (ICls) have revolutionised cancer immunotherapy but their success is wholly dependent on amplifying an existing immune response directed against the tumour. A recent study by Tsuchiya et al. suggests how the properties of induced pluripotent stem cells (iPSCs) may be exploited for the targeted delivery of interferon- $\alpha$ (IFN $\alpha$ ) to elicit an appropriate response.

Recent years have witnessed the renaissance of tumour immunology, driven, in part, by the advent of $\mathrm{ICls}$, monoclonal antibodies specific for inhibitory receptors such as programmed death-ligand 1 (PD-L1) expressed within the tumour microenvironment, the blockade of which releases the brakes on immune responses, maximising their antitumour activity. Success is, however, predicated on the existence of an ongoing immune response to the tumour, which is itself dependent on high genomic instability, responsible for the generation of suitable neoantigens: those tumours that fail to elicit an effective immune response, so-called 'cold' tumours with a low mutational burden, are largely refractory to immune intervention [1]. However, it is not merely amplification of existing immune responses that $\mathrm{ICl}$ seek to achieve but rather their vigorous initiation by dendritic cells (DCs): by altering the balance of stimulatory and inhibitory signals delivered to naïve $T$ cells, the likelihood of initiating an immune response of appropriate magnitude is greatly increased.

But not all DCs are equally effective at eliciting antitumour immunity since the capacity to acquire exogenous tumour-associated antigens (TAAs) and present them via MHC class I, a process known as antigen cross-presentation [2], is a prerequisite for activation of $\mathrm{CD}^{+}$cytotoxic $\mathrm{T}$ lymphocytes (CTLs). This property is confined to rare subsets of DCs that collectively express the chemokine receptor XCR1 [3], responsible for guiding them within the tumour-draining lymph nodes (dLNs) towards $\mathrm{CD}^{+} \mathrm{T}$ cells that secrete the ligand, XCL1. XCR1 ${ }^{+} \mathrm{DC}$ s are surprisingly rare, especially within the tumour itself, which they are known to actively infiltrate. Furthermore, they must undergo maturation before they are able to migrate to the dLNs and elicit a response to TAAs acquired in situ [4]. This process is normally dependent on the sensing of cytosolic DNA via the so-called stimulator of IFN genes (STING) pathway, or the local secretion of potent proinflammatory cytokines, such as IFN $\alpha$, neither of which is abundant in the sterile microenvironment of a solid tumour, representing a significant weak link in antitumour immunity.

Various groups have sought to plug this gap by exploiting the unique properties 